\title{
Pattern of Anti-Microbial Sensitivity and Resistance against Salmonella Species in a Tertiary Hospital in Dhaka
}

\author{
Afsana Begum ${ }^{1}$, Md. Shafiqul Bari ${ }^{2}$, Fazle Rabbi Chowdhury ${ }^{3}$, Naser Ahmed ${ }^{4}$, KAR Sayeed $^{5}$ \\ Received: May 31, 2014 Accepted: March 28, 2015 \\ doi: http://dx.doi.org/10.3329/jemc.v5i2.23378
}

\begin{abstract}
Background: Drug resistant Salmonella spp. continues to remain a health problem as last two decades have witnessed the emergence and spread of multidrug resistance against conventional anti-typhoid drugs. Multidrug resistant (MDR) typhoid is now a serious problem in many developing countries including Bangladesh. Objectives: To find out antimicrobial sensitivity and resistance patterns of different types of Salmonella spp. in patients with enteric fever and to find out the epidemiological strains (e.g. resistant strain, epidemic strain, MDR strain) in patients with enteric fever. Materials and Methods: This cross-sectional study was done during October 2009-November 2010 and January-December 2012 at United Hospital Limited, Dhaka. Laboratory reports of blood culture were collected from those patients who were investigated as suspected cases of enteric fever. Both the indoor and outdoor patients were enrolled in the study. Salmonella species resistant to one or more of the first line drugs were divided into resistant strain, epidemic strain and multi-drug resistant strain. Results: During the period October 2009-November 2010, total 210 subjects were enrolled. Among the participants, 122 were male and 88 were female. S. typhi were found in 133 samples whereas 76 were S. paratyphi A and only 1 S. paratyphi B. Sensitivity was found $77 \%$ for ampicillin, $91.4 \%$ for cotrimoxazole, $78.6 \%$ for chloramphenicol, $87.6 \%$ for ciprofloxacin, $96.6 \%$ for cefixime, $98 \%$ for ceftriaxone and only $22.1 \%$ for azithromycin (77.9\% resistant). Total $91(43.3 \%)$ cases were found having resistant strain, $6(2.8 \%)$ epidemic strain and 8 (3.8\%) MDR strain Salmonella spp. The sensitivity of azithromycin was analyzed among different categories of strains and revealed that $52.7 \%$ showed resistance in resistant strain, $100 \%$ in epidemic strain and $87.5 \%$ in MDR strain. During the period between January-December 2012, total 139 subjects were enrolled. Among the participants 68 were male and 71 were female with a ratio of 1:1.04. S. typhi were found in 84 cases whereas 55 were S. paratyphi A and no S. paratyphi B. Sensitivity was found $62.7 \%$ for ampicillin, $82 \%$ for cotrimoxazole, $83 \%$ for chloramphenicol, $45.2 \%$ for azithromycin, $95 \%$ for ciprofloxacin, 99.3\% for cefixime and 100\% for ceftriaxone. Total 55 (39.6\%) cases were found having resistant strain, $3(2.1 \%)$ epidemic strain and 17 (12.2\%) MDR strain Salmonella spp. Against azithromycin $67.3 \%$ showed resistance in resistant strain, $33.3 \%$ in epidemic strain and $29.4 \%$ in MDR strain. Conclusion: The policy of empirical treatment of enteric fever needs to be rationalized and newer generation antibiotics should be restricted only for multi-resistant cases of enteric fever.
\end{abstract}

Key words: Enteric fever; Salmonella species; Resistance and sensitivity patterns; Multiresistant cases

J Enam Med Col 2015; 5(2): 88-92

1. Associate Consultant, Department of Medicine, United Hospital Limited, Dhaka

2. Associate Professor, Department of Medicine, Sylhet Osmani Medical College, Sylhet

3. Junior Consultant, Department of Medicine, Sylhet Osmani Medical College, Sylhet

4. Classified Specialist, Department of Medicine, Combined Military Hospital, Dhaka

5. Consultant, Department of Microbiology, United Hospital Limited, Dhaka

Correspondence Afsana Begum, Email: afsana_75@yahoo.com 


\section{Introduction}

Enteric fever is a global health problem. Around 21.6 million people are affected (incidence of 3.6 per 1000 population) with enteric fever which kills an estimated 200,000 people every year. The disease is endemic in Indian subcontinent, Southeast Asia, South and Central America and Africa. ${ }^{1}$ Bangladesh being in this region and a country with poor sanitary infrastructure is endemic for enteric fever as other South Asian countries.

Enteric fever has been reported from almost every part of Bangladesh. It is a common cause of febrile illness among the patients presenting to medical facilities and also happens to be the major reason for seeking health service by the general population. Studies conducted in Bangladesh by ICDDR,B showed an incidence of approximately 2000 per 100000 per year. $^{2}$

Chloramphenicol became the standard antibiotic for treating typhoid in $1948 .^{3}$ But in 1972 , chloramphenicol-resistant typhoid fever became a major problem. ${ }^{4}$ Several outbreaks occurred in Mexico, India, Vietnam, Thailand, Korea and Peru. ${ }^{4}$ S. enterica serotype typhi strains were also resistant to sulfonamides, tetracycline and streptomycin, but initially amoxycillin and trimethoprimsulfamethoxazole remained effective alternative drugs. At the end of 1980s and 1990s, S. enterica serotype typhi developed resistance simultaneously to all the first-line drugs (chloramphenicol, trimethoprim, sulfamethoxazole and ampicillin). ${ }^{4}$ Outbreaks of infections with these strains occurred in India, Pakistan, Bangladesh, Vietnam, the Middle East and Africa. ${ }^{5}$

Third generation cephalosporins are now the mainstay of treatment of enteric fever. Isolation of S. typhi resistant to ceftriaxone from Bangladeshi population and reports of clinical strains with higher minimum inhibitory concentration (MIC) from India are increasing. Thus treatment options are getting limited. ${ }^{6,7}$ This situation highlights the need of continued surveillance for changing antibiotic sensitivity and resistance profile of Salmonella spp. over time so that physicians can choose the right antibiotic and prevent misuse of valuable drugs.
This study was conducted to identify the recent epidemiological pattern of Salmonella species in the capital of Bangladesh as well as to analyze the recent trend of antimicrobial sensitivity and resistance against it.

\section{Materials and Methods}

This cross-sectional study was done in the department of Medicine of United Hospital Limited, Dhaka during October 2009-November 2010 and January-December 2012. Laboratory reports of blood culture were collected from those patients who were investigated as suspected cases of enteric fever. Both the indoor and outdoor patients were enrolled in the study. Data were processed and analyzed by rate, ratio and percentage.

Salmonella species resistant to one or more of the first line drugs (chloramphenical, cotrimoxazole and ampicillin) were divided into resistant strain, epidemic strain and multi-drug resistant strain. ${ }^{8}$ Resistant strains of Salmonella were resistant to at least one of the first line drugs. Epidemic strains of Salmonella were resistant to chloramphenicol and cotrimoxazole but susceptible to ampicillin. Multidrug-resistant S. typhi (MDRST) is mainly reserved for strains resistant to all three first-line antityphoidal antimicrobial agents, namely ampicillin, chloramphenicol and cotrimoxazole. ${ }^{9}$

\section{Results}

During the period October 2009-November 2010, total 210 subjects were enrolled. Among the participants, 122 were male and 88 were female with a ratio of 1.3:1. During the period JanuaryDecember 2012, total 139 subjects were enrolled. Among the participants 68 were male and 71 were female with a ratio of 1:1.04. Age distribution of culture positive enteric fever subjects is shown in Fig 1. S. typhi was found in 133 cases followed by 76 cases of S. paratyphi A and only $1 \mathrm{~S}$. paratyphi B in the period of October 2009-November 2010 (Fig 2). S. typhi was found in 84 cases followed by 55 cases of S. paratyphi A and no S. paratyphi B in the period of January-December 2012 (Fig 2). 


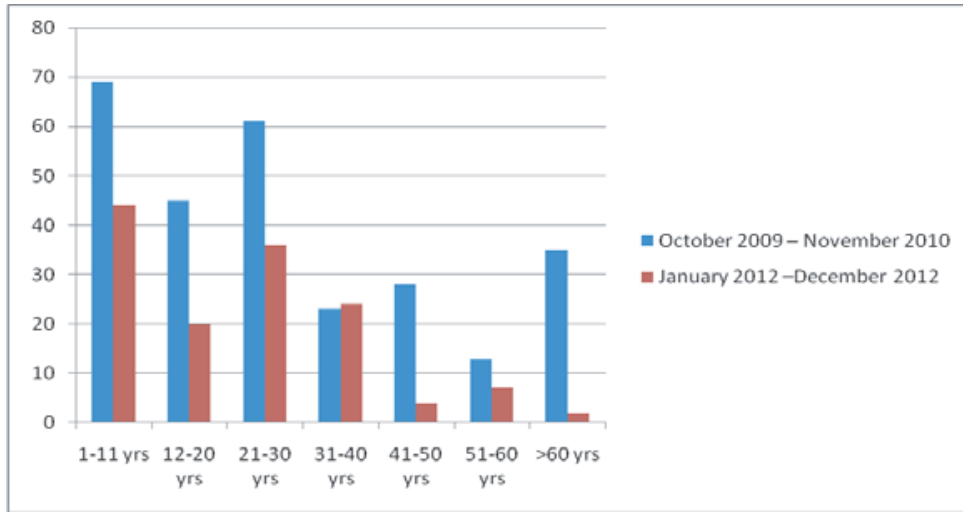

Fig 1. Age distribution of culture positive enteric fever subjects

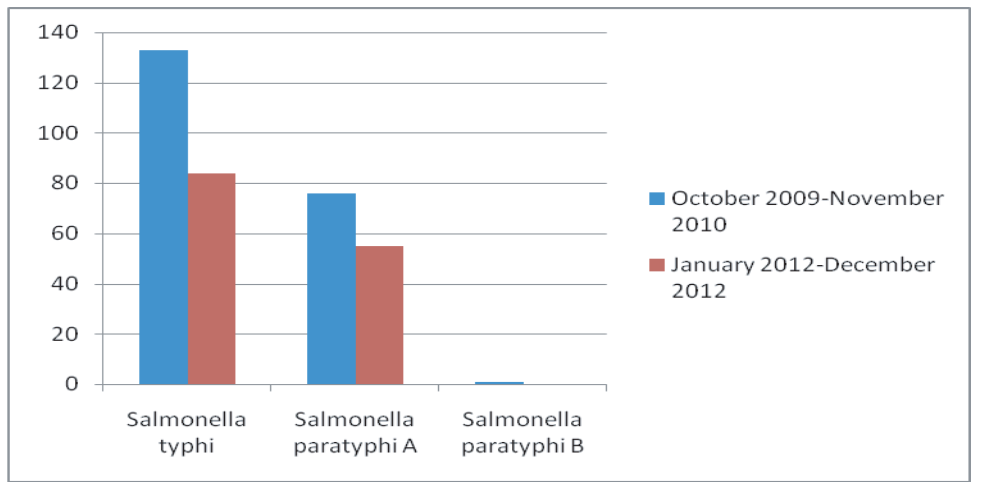

During the period October 2009-November 2010 , sensitivity was found $77 \%$ for ampicillin, $91.4 \%$ for cotrimoxazole, $78.6 \%$ for chloramphenicol, $87.6 \%$ for ciprofloxacin, $96.6 \%$ for cefixime, $98 \%$ for ceftriaxone and only $22.1 \%$ for azithromycin (77.9\% resistant) (Table I). During this period $91(43.3 \%)$ cases were found having resistant strain, $6(2.8 \%)$ epidemic strain and $8(3.8 \%)$ MDR strain Salmonella spp. (Table II). During the period January-December 2012, sensitivity was found $62.7 \%$ for ampicillin, $82 \%$ for cotrimoxazole, $83 \%$ for chloramphenicol, $45.2 \%$ for azithromycin, $95 \%$ for ciprofloxacin, $99.3 \%$ for cefixime and $100 \%$ for ceftriaxone (Table I). During this period, there were $55(39.6 \%)$ resistant strain, $3(2.1 \%)$ epidemic strain and 17 (12.2\%) MDR strain Salmonella spp. (Table II).

Fig 2. Distribution of isolated organisms

Table I: Antimicrobial sensitivity pattern in enteric fever

\begin{tabular}{|l|c|c|l|r|r|}
\multicolumn{4}{|c|}{ October 2009-November 2010} & \multicolumn{3}{|c|}{ January 2012-December 2012} \\
\hline Drugs & Sensitive (\%) & Resistant $(\%)$ & Drugs & Sensitive $(\%)$ & Resistant $(\%)$ \\
\hline Ciprofloxacin $(\mathrm{n}=209)$ & $183(87.6)$ & $26(12.4)$ & Ciprofloxacin $(\mathrm{n}=139)$ & $132(95)$ & $7(5)$ \\
\hline Cotrimoxazole $(\mathrm{n}=209)$ & $191(91.4)$ & $18(8.6)$ & Cotrimoxazole $(\mathrm{n}=139)$ & $114(82)$ & $25(18)$ \\
\hline Chloramphenicol $(\mathrm{n}=206)$ & $162(78.6)$ & $44(21.4)$ & Chloramphenicol $(\mathrm{n}=135)$ & $112(83)$ & $23(17)$ \\
\hline Cefixime $(\mathrm{n}=203)$ & $196(96.6)$ & $7(3.4)$ & Cefixime $(\mathrm{n}=139)$ & $138(99.3)$ & $1(0.7)$ \\
\hline Ampicillin $(\mathrm{n}=200)$ & $154(77)$ & $46(23)$ & Ampicillin $(\mathrm{n}=134)$ & $84(62.7)$ & $50(37.3)$ \\
\hline Azithromycin $(\mathrm{n}=204)$ & $45(22.1)$ & $159(77.9)$ & Azithromycin $(\mathrm{n}=135)$ & $61(45.2)$ & $74(54.8)$ \\
\hline Ceftriaxone $(\mathrm{n}=205)$ & $201(98)$ & $4(2)$ & Ceftriaxone $(\mathrm{n}=139)$ & $139(100)$ & $0(0)$ \\
\hline
\end{tabular}

Table II: Epidemiologic resistant strain pattern in enteric fever

\begin{tabular}{|l|c|c|c|c|}
\hline \multicolumn{1}{|c|}{ Epidemiologic strains } & \multicolumn{2}{|c|}{ Oct 2009-Nov 2010 } & \multicolumn{2}{c|}{ Jan-Dec 2012 } \\
\hline & Frequency & Percentage & Frequency & Percentage \\
\hline Resistant strain & 91 & 43.3 & 55 & 39.6 \\
\hline Epidemic strain & 6 & 2.8 & 3 & 2.1 \\
\hline Multi-drug resistant strain & 8 & 3.8 & 17 & 12.2 \\
\hline
\end{tabular}


Table III: Pattern of azithromycin resistance among various epidemiological strains

\begin{tabular}{|l|c|c|}
\hline \multicolumn{3}{|c|}{ October 2009-November 2010 } \\
\hline Epidemiologic strains & Frequency & $\%$ \\
\hline Resistant $(n=91)$ & 48 & 52.7 \\
\hline Epidemic $(n=6)$ & 6 & 100 \\
\hline Multidrug resistrant $(n=8)$ & 7 & 87.5 \\
\hline
\end{tabular}

The sensitivity to azithromycin was analyzed among different strains. During the period between October 2009-November 2010, 52.7\% showed resistance in resistant strain, $100 \%$ in epidemic strain and $87.5 \%$ in MDR strain (Table III). During the period between January-December 2012, 67.3\% showed resistance in resistant strain, $33.3 \%$ in epidemic strain and $29.4 \%$ in MDR strain (Table III).

\section{Discussion}

Typhoid fever is highly endemic in developing countries like Bangladesh and is an important cause of health problem involving huge health care costs, high morbidity and economic loss. Children are affected mostly between 5-12 years. ${ }^{10}$ Atypical presentation of typhoid fever may be misdiagnosed in pre-school age group due to an underdeveloped reticuloendothelial system. ${ }^{11}$

In our study causative organisms of enteric fever were predominantly $\mathrm{S}$. typhi followed by $\mathrm{S}$. paratyphi. Shirakawa et al found S. paratyphi as the commonest cause of typhoid fever. ${ }^{12}$

In this study Salmonella spp. remained sensitive to chloramphenicol, ampicillin, and cotrimoxazole over both study periods. The re-emergence of increased sensitivity of cotrimoxazole and chloramphenicol to Salmonella should be considered in using it as first line therapy. High susceptibility to chloramphenicol, cotrimoxazole, and ampicillin were also reported from several parts of India such as Bangaluru, Chennai, Chandigarh, central west, and northern India. ${ }^{13} \mathrm{~A}$ study done by Chand et al ${ }^{14}$ revealed reemergence of susceptibility to ampicillin, chloramphenicol and cotrimoxazole in great proportion.

Patients in Indian subcontinent or with the history of travel to the Indian subcontinent were suggested to
January-December 2012

\begin{tabular}{|l|c|c|}
\hline Epidemiologic strains & Frequency & $\%$ \\
\hline Resistant $(\mathrm{n}=55)$ & 37 & 67.3 \\
\hline Epidemic $(\mathrm{n}=3)$ & 1 & 33.3 \\
\hline Multidrug resistant $(\mathrm{n}=17)$ & 5 & 29.4 \\
\hline
\end{tabular}

receive ciprofloxacin as first line therapy. ${ }^{15}$ But S. typhi isolates resistant to ciprofloxacin and ceftriaxone have been reported. S. typhi strains with reduced susceptibility to fluoroquinolones have become a major problem in Asia and other parts of the world. ${ }^{16}$ In this study, sensitivity to ciprofloxacin increased from the first study period $(87.6 \%)$ to the second $(95 \%)$.

Optimal antimicrobial treatment of patients with enteric fever depends on an understanding of local patterns of antimicrobial resistance and is enhanced by the results of antimicrobial susceptibility testing of the Salmonella isolated from the individual patient. Third-generation cephalosporins such as ceftriaxone may be used. However, the cost and route of administration make ceftriaxone less suitable for treatment in some low- and middleincome countries, and the oral third-generation cephalosporin cefixime appears to be inferior to other oral agents both in terms of fever clearance time and treatment failure. ${ }^{17}$ In this study, sensitivity to ceftriaxone was high in both periods. All the 139 isolates of January-December 2012 were sensitive to ceftriaxone which correlates with the study of Arora et al. ${ }^{18}$ This underlies the importance of the drug for treating MDR and ciprofloxacin resistant enteric fever cases. Emphasis has to be laid on the sparing use of the drug to prevent the occurrence of resistance to ceftriaxone.

WHO recommends the fluoroquinolones or cefixime for the treatment of MDR typhoid fever and azithromycin, the third-generation cephalosporins, or a 10-14 day course of high-dose older generation fluoroquinolones for the treatment of nalidixic acid resistant typhoid (NART). But in this study resistance of different strains of Salmonella to azithromycin is very high. 
Antibiotics are unnecessarily prescribed for infections such as the common cold, cough and diarrhea which are usually of viral etiology and can be resolved by the immune system. Emphasis is placed on treatment instead of finding the causative organism and reaching a proper diagnosis. This leads to patients being treated with broad spectrum antibiotics, which results in the emergence of MDR organisms. ${ }^{15}$

A limitation of our study was that clinical outcomes were not analyzed. Quinolones may remain effective despite in vitro resistance and ceftriaxone may be associated with prolonged time to fever resolution despite in vitro sensitivity.

The policy of empirical treatment of enteric fever needs to be rationalized and newer generation antibiotics should be used only for multi-resistant cases of enteric fever. Consistent surveillance and antibiotic susceptibility testing is required in different parts of the country to know the sensitivity pattern among enteric fever pathogens, which will be helpful for clinicians for rational prescribing.

\section{References}

1. Eric Mintz. Enteric fever. Epidemiology and reports from the field: global situation and WHO recommendations. In: Proceedings of the $8^{\text {th }}$ International Conference Asia Pacific symposium on typhoid fever and other Salmonellosis in Dhaka, Bangladesh, 2013.

2. Background document: the diagnosis, treatment and prevention of typhoid fever, World Health Organization, 2003.

3. Woodward TE, Smadel JE, Ley HL Jr, Green R, Mankikar DS. Preliminary report on the beneficial effect of chloromycetin in the treatment of typhoid fever. Ann Intern Med 1948; 29: 131-134.

4. Mirza SH, Beeching NJ, Hart CA. Multi-drug resistant typhoid: a global problem. J Med Microbiol 1996; 44: 317-319.

5. Hermans PWM, Saha SK, van Leeuwen WJ, Verbrugh HA, van Belkum A, Goessens WHF. Molecular typing of Salmonella typhi strains from Dhaka (Bangladesh) and development of DNA probes identifying plasmid-encoded multidrug-resistant isolates. J Clin Microbiol 1996; 34: 1373-1379.

6. Saha SK, Talkukder SY, Islam M, Saha S. A highly ceftriaxone resistant salmonella typhi in Bangladesh. Peadiatr Infect Dis J 1999; 18: 387.
7. Capoor MR, Nair D, Hasan AS, Aggarwal P, Gupta B. Typhoid fever: narrowing therapeutic options in India. Southeast Asian J Trop Med Public Health 2006; 37: 1170-1174.

8. Pato-Mesola VV, Donaldo MES. Antimicrobial susceptibility of salmonella typhi isolates from government and private hospitals in Cebu city. Phil J Microbiol Infect Dis 1997; 26(1): 5-8.

9. Muhammad AM. What after ciprofloxacin and ceftriaxone in treatment of Salmonella typhi. Pak J Med Sci 2006; 22(1): 51-54.

10. Siddiqui FJ, Rabbani F, Hasan R, Nizami SQ, Bhutta ZA. Typhoid fever in children: some epidemiological considerations from Karachi, Pakistan. Int J Infect Dis 2006; 10: 215-222.

11. Sinha A, Sazawal S, Kumar R, Sood S, Reddaiah VP, Singh B et al. Typhoid fever in children aged less than 5 years. Lancet 1999; 354: 734-737.

12. Shirakawa T, Acharya B, Kinoshita S, Kumagai S, Gotoh A, Kawabata M. Decreased susceptibility to fluoroquinolones and gyrA gene mutation in the Salmonella enterica serovar Typhi and Paratyphi A isolated in Kathmandu, Nepal in 2003. Diagn Microbiol Infect Dis 2006; 54: 299-303.

13. Kumar Y, Sharma A, Mani KR. Re-emergence of susceptibility to conventionally used drugs among strains of Salmonella Typhi in central west India. J Infect Dev Ctries 2011; 5(3): 227-230.

14. Chand HJ, Rijal KR, Neupane B, Sharma VK, Jha B. Reemergence of susceptibility to conventional first line drugs in Salmonella isolates from enteric fever patients in Nepal. J Infect Dev Ctries 2014; 8(11): 1483-1487.

15. Rowe B, Ward LR, Threlfall EJ. Ciprofloxacin-resistant Salmonella typhi in the UK. Lancet 1995; 346: 1302.

16. Crump JA, Barrett TJ, Nelson JT, Angulo FJ. Reevaluating fluoroquinolone breakpoints for Salmonella enterica serotype Typhi and for non-Typhi salmonellae. Clin Infect Dis 2003; 37: 75-81.

17. Crump JA, Mintz ED, Hughes JM, Wilson ME. Global trends in typhoid and paratyphoid fever. Clinical Infectious Diseases; 50(2): 241-246.

18. Arora D, Gupta P, Gill G, Chawla R, Singla R. Changing trends in the antibiograms of salmonella isolates in northern area of Punjab. International Journal of Pharmacy and Pharmaceutical Sciences 2010; 2(Suppl 3): 135-137.

19. Zaki SA, Karande S. Multi-drug resistant typhoid fever, a review. J Infect Dev Ctries 2011; 5(5): 324-337. 